Check for updates

Cite this: RSC Adv., 2018, 8, 41323

Received 3rd August 2018

Accepted 20th November 2018

DOI: $10.1039 / \mathrm{c} 8 \mathrm{ra06531d}$

rsc.li/rsc-advances

\section{Facile synthesis of an all-in-one graphene nanosheetsanickel electrode for high-power performance supercapacitor application $\uparrow$}

\author{
Bing Huang, (D) *a Zhiyuan Zhao, (D) *a Jian Chen, ${ }^{a}$ Yuzhen Sun, ${ }^{a}$ Xiaowei Yang, ${ }^{a}$ \\ Jian Wang, ${ }^{\text {ab }}$ Hao Shen ${ }^{\mathrm{b}}$ and Ye Jin ${ }^{\mathrm{b}}$
}

\begin{abstract}
We report a facile and novel approach for the fabrication of all-in-one supercapacitor electrodes by in situ electrochemical exfoliation of flexible graphite paper (FGP) on a nickel collector. The binder-free three dimensional (3D) graphene nanosheets@Ni (GNSs@Ni) supercapacitor electrodes exhibit a high specific capacitance of $196.4 \mathrm{~F} \mathrm{~g}^{-1}$ and $36.2 \mathrm{mF} \mathrm{cm}^{-2}$, respectively, at a scan rate of $50 \mathrm{mV} \mathrm{s}^{-1}$. Even at the high scan rate of $2500 \mathrm{mV} \mathrm{s}^{-1}$ the specific capacitance of the capacitor still shows a retention of $85.6 \%(168 \mathrm{~F}$ $\mathrm{g}^{-1}, 31 \mathrm{mF} \mathrm{cm}{ }^{-2}$ ). Meanwhile, the as-prepared electrode offers excellent cycling performance with $91.5 \%$ capacitance retention after 100000 charging-discharging cycles even at the high current density of $11 \mathrm{~A} \mathrm{~g}^{-1}$. Such high rate capability, specific capacitance and exceptional cycling ability of the GNSsaNi electrode are attributed to the all-in-one architecture which provides unique properties including high electrical conductivity, large specific surface area and excellent electrochemical stability. We anticipate that these results will shed light on new strategies to synthesize high-performance hybrid nanoarchitectures electrodes using the prepared graphene nanosheets as the support, which offers great potential in energy storage devices and electrochemical catalysis applications.
\end{abstract}

\section{Introduction}

Energy and the environment are two major themes of human development nowadays. To solve the energy problem encountered in modern society, the demand for breakthroughs in battery or supercapacitor science and technology is continuously getting higher. ${ }^{1-5}$ Supercapacitors, also known as electric double-layer capacitors (EDLCs), which have the advantages of both conventional dielectric capacitors and batteries, have attracted tremendous attention because they can provide greater energy density than conventional capacitors, and higher power density and longer cycle life than batteries. They are expected to be broadly used in portable electronics, space systems and electric vehicles etc. ${ }^{6-8}$ EDLCs achieve the storage of energy through the pure electric charge adsorbed at the interface between a carbon electrode and electrolyte. The performance of EDLCs is mainly determined by the properties of the carbon materials, such as the specific surface area, conductivity and

${ }^{a}$ Institute of New Energy on Chemical Storage and Power Sources, College of Applied Chemistry and Environmental Engineering, Yancheng Teachers University, Yancheng, 224000, China. E-mail: huangbingbzu@sina.com; zhiyuan.zhao@rcclab. com; Fax: +86515 68665372; Tel: +8651568665372

${ }^{b}$ College of Chemistry and Chemical Engineering, Nanjing Tech University, Nanjing, 210009, China

$\dagger$ Electronic supplementary information (ESI) available. See DOI: 10.1039/c8ra06531d porosity., ${ }^{\mathbf{9} 10}$ To date, many reports have focused on different forms of carbon-based materials, e.g., activated carbons (ACs), ${ }^{11-13}$ carbon nanotubes (CNTs), ${ }^{14-16}$ mesoporous carbon, ${ }^{17-20}$ carbon nanofibers, ${ }^{21}$ or carbide-derived carbons (CDCs), ${ }^{22}$ to fabricate the carbon electrodes of EDLCs, but few carbon materials can show excellent performance in all three aspects. For example, since the micropores in activated carbon hinder the formation of double layers, and the wetting efficiency of electrolytes, AC-based capacitors only achieve about $10-20 \%$ of their theoretical capacitance. ${ }^{23}$ Therefore, great efforts have been devoted to design new materials or electrodes to enhance the energy storage performance of carbon-based EDLCs. Graphene has an extraordinarily large surface area (2675 $\mathrm{m}^{2} \mathrm{~g}^{-1}$ ), excellent electrical and thermal conductivity, which makes it an ideal electrode material for supercapacitors. ${ }^{24-26}$ As for conventional slurry-derived electrodes, the non-uniform distribution of aggregated graphene materials reduce the effective capacitance of the electrodes and nonconductive adhesives severely suppress the intrinsically high conductivity of the supercapacitor. To prevent self-aggregation and restacking of graphene sheets, one promising approach is to develop three dimensional (3D) graphene architectures as supercapacitor electrodes. ${ }^{27-32}$ To reduce the contact resistance between graphene materials and the current collector for supercapacitor with high power characteristics, the common strategy is the fabrication of binder-free supercapacitor electrodes such as graphene paper electrodes prepared by the flow- 
directed assembly of individual GO/graphene sheets. ${ }^{33-35}$ However, the problem remains that the barrier between graphene and the electrolyte reduces the conductivity of graphene materials due to the closely stacked structure of graphene paper. Therefore, it is desired to develop the fabrication of electrodes of supercapacitor with excellent performance using binder-free 3D graphene electrodes, especially using a simple technique.

In this work, we propose a simple in situ electrochemical exfoliation approach to fabricate binder-free 3D graphene nanosheets@Ni (GNSs@Ni) electrodes, in which 3D graphene nanosheets are used as the active material and nickel metal acts as current collector. On the one hand, the graphene nanosheets are rooted on the surface of the nickel matrix, which makes the binder-free contact resistance between GNSs and the Ni current collectors extremely low and the electrode structure is robust. On the other hand, GNSs fastened with metal nickel prevents them from agglomerating. Therefore, the GNSs fastened on the Ni matrix show excellent high rate capability, cyclic stability and high specific capacitance.

\section{Experimental}

\subsection{Electrode materials preparation}

The fabrication process of GNSs@Ni supercapacitor electrode is illustrated in Fig. 1. Firstly, the FGP@Ni electrode (Fig. 1b and f) was prepared by electrochemical deposition. Electroplating was carried out in self-prepared nickel plating solution by FGP (Fig. 1a and d, $0.05 \mathrm{~mm}$ thickness, Shenzhen DUBANG science and technology Co., Ltd.) as cathode and nickel plate as anode (Fig. 1e). Then, GNSs@Ni electrode was prepared via electrochemical exfoliation approach. FGP electroplated with nickel was utilized as anode electrode and a Pt plate was employed as a counter electrode. The FGP@Ni and Pt plate were placed in the electrolytic cell, with $10 \mathrm{M}$ sulfuric acid aqueous solution as electrolyte. A multi-impulse potential of $2 \mathrm{~V}$ for $20 \mathrm{~s}$ and $10 \mathrm{~V}$ for
$10 \mathrm{~s}$ was applied to the FGP@Ni electrode for 10 cycles at room temperature. When the $2 \mathrm{~V}$ voltage was applied to the FGP/Ni electrode, the color of the FGP became blue (Fig. 1g), which indicated the formation of $\mathrm{H}_{2} \mathrm{SO}_{4}$-graphite intercalation compound (GIC), ${ }^{25}$ followed by ramping the potential to $10 \mathrm{~V}$ voltage, the surface of the electrode became brown accompanied by a large number of bubbles (Fig. 1h). Finally, the excess carbon on the surface fell off, and the GIC fastened on the nickel matrix were electrochemical exfoliated into GNSs (Fig. 1c, $\mathrm{i}$ and $\mathrm{j}$ ).

\subsection{Physicochemical characterization}

The morphology and microstructure of the GNSs@Ni samples were characterized by field-emission scanning electron microscopy (FESEM, Hitachi S-4800) equipped with an X-ray energy dispersive spectrometer (EDS) and transmission electron microscopy (TEM, JEM-2100). The crystallographic structures of the samples were determined using X-ray diffraction spectroscopy (XRD, Ultima-IV, Rigaku) equipped with $\mathrm{Cu} \mathrm{K} \alpha$ radiation source at a scan rate of $8^{\circ} \mathrm{min}^{-1}$ from $10^{\circ}$ to $80^{\circ}(2 \theta$ degree). Raman spectroscopy (Lab RAMHR, Horiba, Jobin Yvon, $532 \mathrm{~nm}$ laser excitation) was performed to characterize the microstructure of the samples. The content of carbon elements in the GNSs@Ni and FGP@Ni electrodes was determined precisely by high frequency infrared carbon and sulfur analyzer (HW2000B, YingZhiCheng, Wuxi, China), and it is $0.18 \mathrm{mg} \mathrm{cm}^{-2}$ and $5.2 \mathrm{mg} \mathrm{cm}^{-2}$, respectively.

\subsection{Electrochemical characterization}

The electrochemical performance of the working electrode (WE), i.e., GNSs@Ni electrode, was evaluated using a threeelectrode configuration in the voltage window between -0.15 and $0.3 \mathrm{~V}$, while the other side of Ni was sealed using $50 \%$ SBR glue. Pt foil and mercury/mercury oxide electrode were used as counter electrodes (CE) and reference electrode (RE) respectively. The electrolyte including $6 \mathrm{M} \mathrm{KOH}$ was used in the

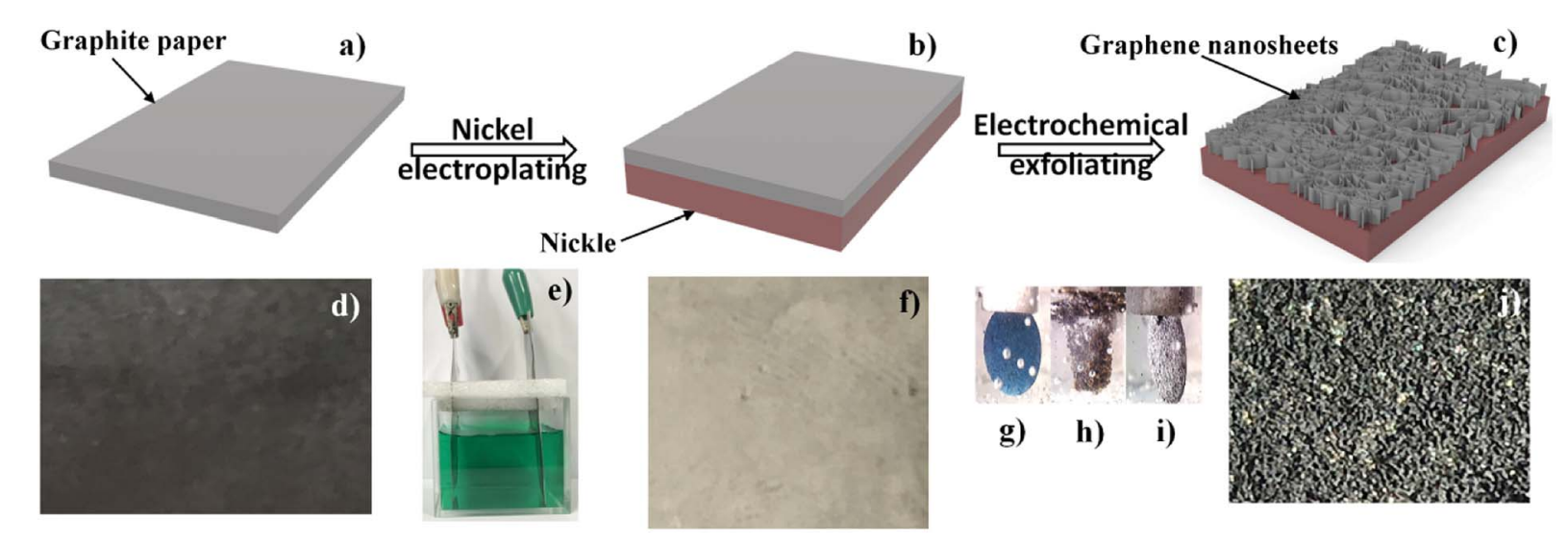

Fig. 1 Schematic illustration of the preparation procedure of GNSs(aNi electrode. A flexible graphite paper (a) (the photograph is shown in (d)) was coated by nickel to form FGP@Ni electrode (b) using electroplating (the photograph is shown in (f)). Then graphene nanosheets (c) (the photograph is shown in (j)) were created via electrochemical exfoliation approach to form GNSs@Ni electrode. (e) Graphite paper as cathode and nickel plate as anode during electroplating; $(\mathrm{g})$ when the $2 \mathrm{~V}$ voltage was applied to the FGP/Ni electrode, the color of the FGP became blue; ( $h$ ) with ramping the potential to $10 \mathrm{~V}$ voltage, the surface of the electrode became brown accompanied by a large number of bubbles; (i) the excess carbon on the surface fell off, and GNSs was formed. 

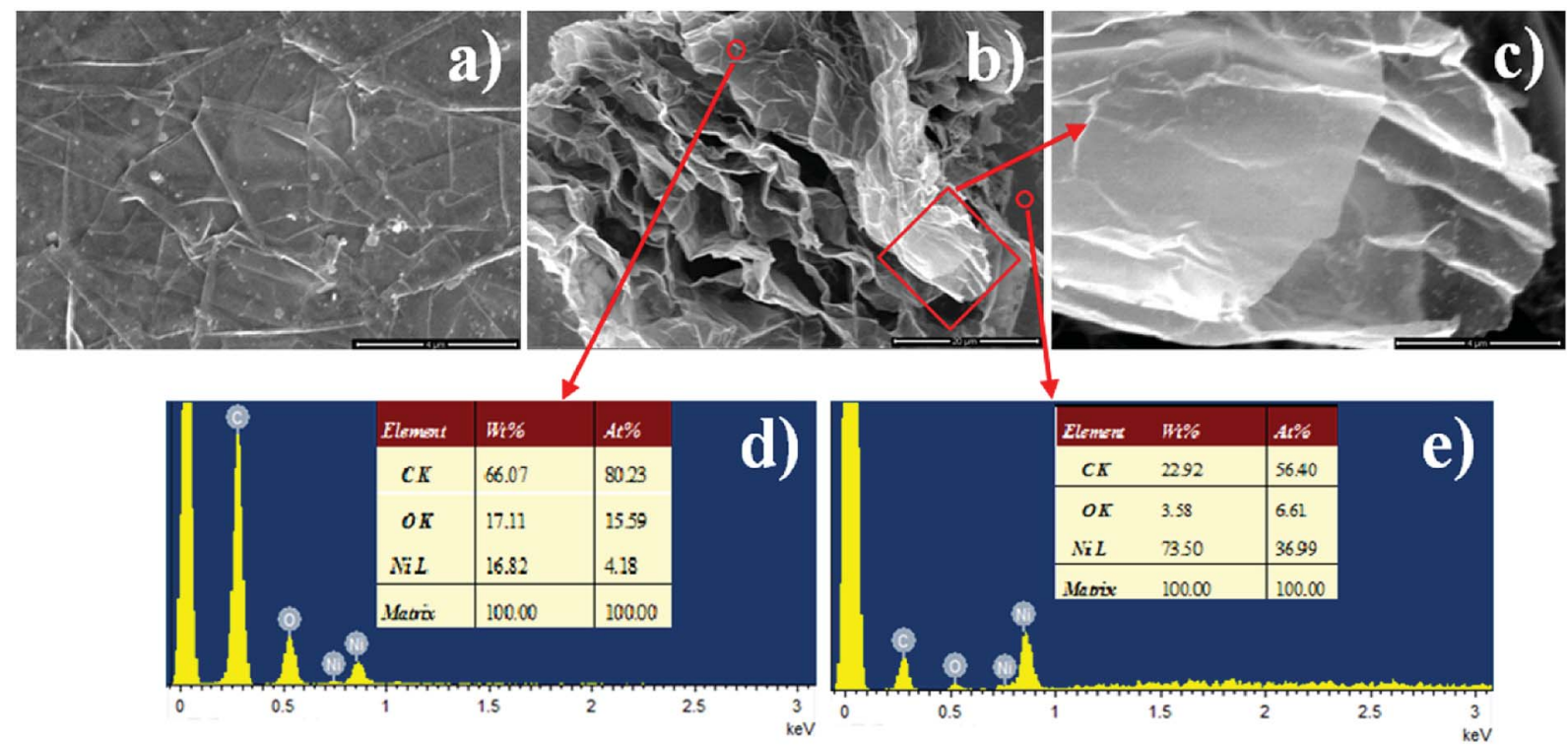

Fig. 2 SEM images of FGP (a), electrochemical exfoliated GNSs with low (b) and high magnification (c), different areas EDS spectrums of the asprepared GNSs@Ni electrode (d and e).

current work. Cyclic voltammetry (CV) and electrochemical impedance spectroscopy (EIS) with the frequency ranging from $0.1 \mathrm{~Hz}$ to $100 \mathrm{kHz}$ were conducted on a electrochemical workstation (CHI 760E, Shanghai Chenhua). Galvanostatic charge/ discharge (GCD) measurements were carried out by a chargedischarge unit (Neware, Shenzhen, China).

\section{Results and discussion}

As the transformation of the original FGP into GNSs is a crucial step that determines the performance of supercapacitor electrodes, we first demonstrate that the electrochemically exfoliated GNSs were successfully fabricated by FESEM (Fig. 2). As shown in Fig. 2a, it is clear to see the smooth surface of FGP, on which the small graphite flakes are visible. In contrast, the resulting three-dimensional multilayer GNSs after electrochemically exfoliation show much different morphology from FGP (Fig. 2b, and S1 $\dagger$ ). GNSs, perpendicular to the surface of nickel substrate, are fastened to the nickel matrix and the root segments of the graphene go deep into the metal matrix to form an ideal conductive network structure, which will promote electrochemical reactions and electron transfer. A magnified image of GNSs shows that the surface of the pristine GNSs are very clean and wrinkled (Fig. 2c). Therefore, the highly fluffy structure and curved thin flaky appearance are suitable for supercapacitors applications. The EDS images (Fig. 2d and e) of the selected region in Fig. $2 \mathrm{~b}$ confirms that carbon content of GNSs is 80.23 at\% higher than the 56.4 at\% of the nickel matrix. The carbon on the nickel matrix mainly comes from the surface of FGS and it is bound by the bond force of $\mathrm{Ni}-\mathrm{C}$ in the process of electroplating. The structure of the carbon embedded in the nickel matrix can reduce the resistance of the GNSs@Ni electrode and avoid the restacking of graphene nanosheets due to van der Waals interactions.
TEM was employed to further characterize the graphene nanosheets scraped from the surface of the nickel matrix. As shown in Fig. 3a, the transparent and folded exfoliated graphene nanosheet has a clean surface and is free of contaminants. Furthermore, the hexagonal crystalline structure of multi-layer graphene nanosheet has been confirmed by the sixfold symmetry pattern of selected area electron diffraction (SAED) of the graphene nanosheet (Fig. 3b). ${ }^{36,37}$

To study graphite and graphene materials, Raman spectroscopy is usually used to analyze their electronic structure and defect concentration. As is well-known, there are two characteristic peaks, named $\mathrm{D}$ band and $\mathrm{G}$ band in carbon-based materials. The D band corresponds to the j-point photons of $\mathrm{A}_{1 \mathrm{~g}}$ symmetry or breathing mode, related to the disorder band of a structural defect, such as amorphous carbon, or an edge. ${ }^{38}$ While the $\mathrm{G}$ band represents the first-order scattering of the $\mathrm{E}_{2 \mathrm{~g}}$ photons in the $\mathrm{sp}^{2}$ carbon domain. Thus, the intensity ratio of the $\mathrm{D}$ band to $\mathrm{G}$ band $\left(I_{\mathrm{D}} / I_{\mathrm{G}}\right)$ is usually used to represent the disorder degree of carbon materials. As shown in Fig. 4, the FGP exhibits characteristic D band at $1352 \mathrm{~cm}^{-1}$ and $\mathrm{G}$ band at $1583 \mathrm{~cm}^{-1}$ (the black line in Fig. 4), while the spectra of electrochemical exfoliated GNSs (the red line in Fig. 4) shows a D band at $1347 \mathrm{~cm}^{-1}$, and a $\mathrm{G}$ band at $1582 \mathrm{~cm}^{-1}$. After electrochemical exfoliation, there is a notable increase in $I_{\mathrm{D}} / I_{\mathrm{G}}$ ratio for GNSs compared to FGP (from 0.13 to 1.23), which reveals that the electrochemical oxidation and the exfoliation of graphene layers reduce the degree of order of the graphite crystal structure and the size of in-plane $\mathrm{sp}^{2}$ domains. ${ }^{39}$ It is well-known that the width, position and shape of the $2 \mathrm{D}$ peak are very sensitive to the number of graphene layers. For FGP, the $2 \mathrm{D}$ peak is at $2718 \mathrm{~cm}^{-1}$ and it could be fitted as two Lorentz peaks. GNSs, however, show a symmetric $2 \mathrm{D}$ peak with a red shift to $2694 \mathrm{~cm}^{-1}$, indicating that multi-layer graphene sheets are successfully exfoliated. ${ }^{40}$ 

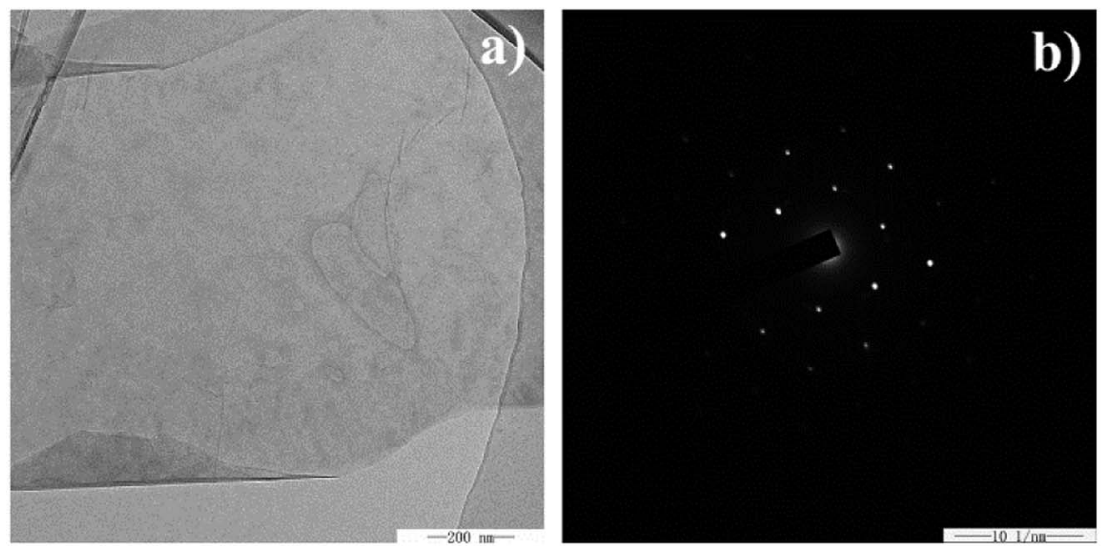

Fig. 3 Typical TEM image for GNSs (a), SAED pattern of GNSs (b).

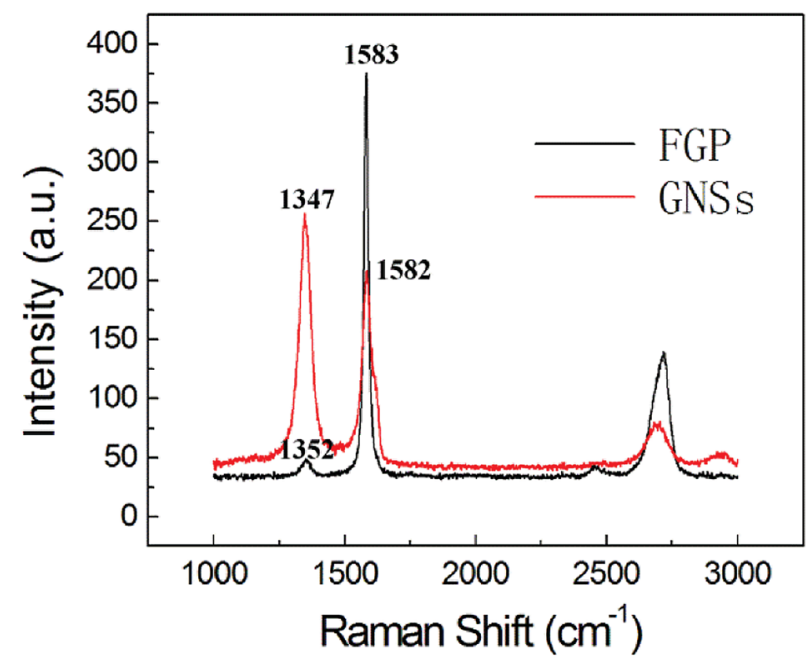

Fig. 4 Raman spectra of FGP@Ni and GNSs@Ni electrodes.
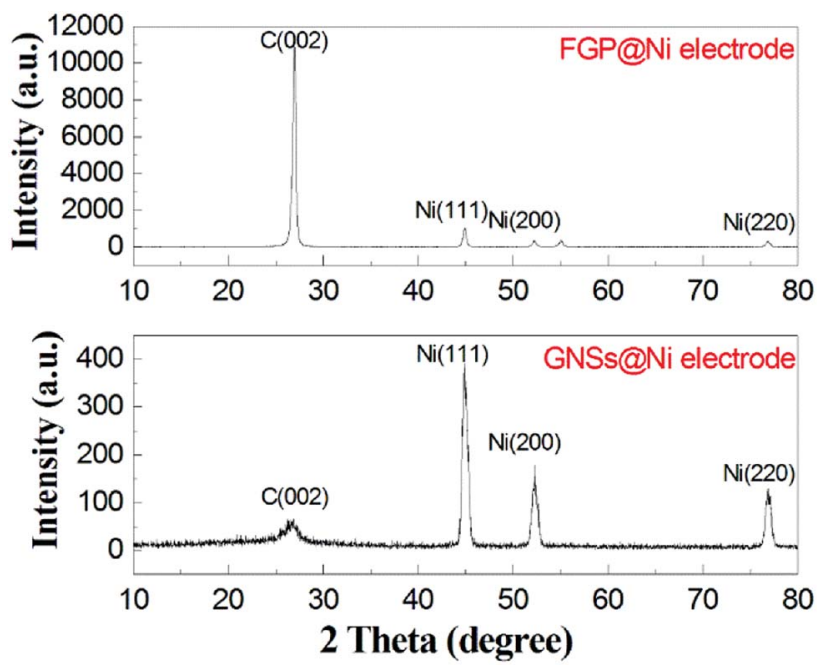

Fig. 5 XRD pattern of the FGP@Ni and GNSs@Ni electrodes.
As shown in Fig. 5, the crystal structure of the nickel-fastened graphene nanosheets was analyzed by XRD. FGP has a high intensity characteristic peak at $26.6^{\circ}$, however, for GNSs, this characteristic peak (002) is prominently weakened, and broadened compared with that of the FGP. This XRD result demonstrates that the long-range periodicity associated with the $c$ axis in the thick FGP is modified. ${ }^{25}$

To understand the role of the all-in-one architecture (in situ formation of 3D graphene nanosheets on the surface of nickel matrix) of the GNSs@Ni electrode on its electrochemical properties, the specific power, specific capacitance and cycle stability tests were performed. As a comparison, the electrochemical performance of the original FGP@Ni was also tested in the same condition. $\mathrm{CV}$ was used to monitor the electrochemical behavior and properties of the two supercapacitors using GNSs@Ni and FGP@Ni as electrodes at scan rates varying from 50 to $2500 \mathrm{mV} \mathrm{s}^{-1}$. As shown in Fig. 6, the $\mathrm{CV}$ profiles of the GNSs@Ni electrode (Fig. 6a) are close to the ideal rectangular shape, confirming fast charge propagation within the electrodes and the formation of an efficient EDL capacitor. In addition, the $\mathrm{CV}$ remains rectangular in shape even at a fast scan rate of 2500 $\mathrm{mVs}^{-1}$, indicating that the high-power capability could be attributed to the small internal resistance in the electrode. In comparison, the CV curves of the FGP@Ni (Fig. 6b) electrode obviously deviate from the standard rectangular shape, especially at a relatively high scan rate. Although FGP is a highly conductive substrate, expanded graphite sheets compacted in FGP have small porosity and contact area with the electrolyte. After the electrochemical exfoliation, graphene nanosheets have more surfaces and exposed edge defects, which may increase the affinity with electrolyte through the capillary interaction. Meanwhile, the interconnection of graphene nanosheets and nickel matrix would enhance the conductivity of the GNSs@Ni electrode. The area current density of GNSs@Ni electrode is one order of magnitude higher than that of the FGP@Ni electrode, and the mass current density is 400 times that of the FGP@Ni electrode (Fig. 6a and b) indicating the significantly enlarged surface area (obtained by electrochemical exfoliation) available for charge storage. 

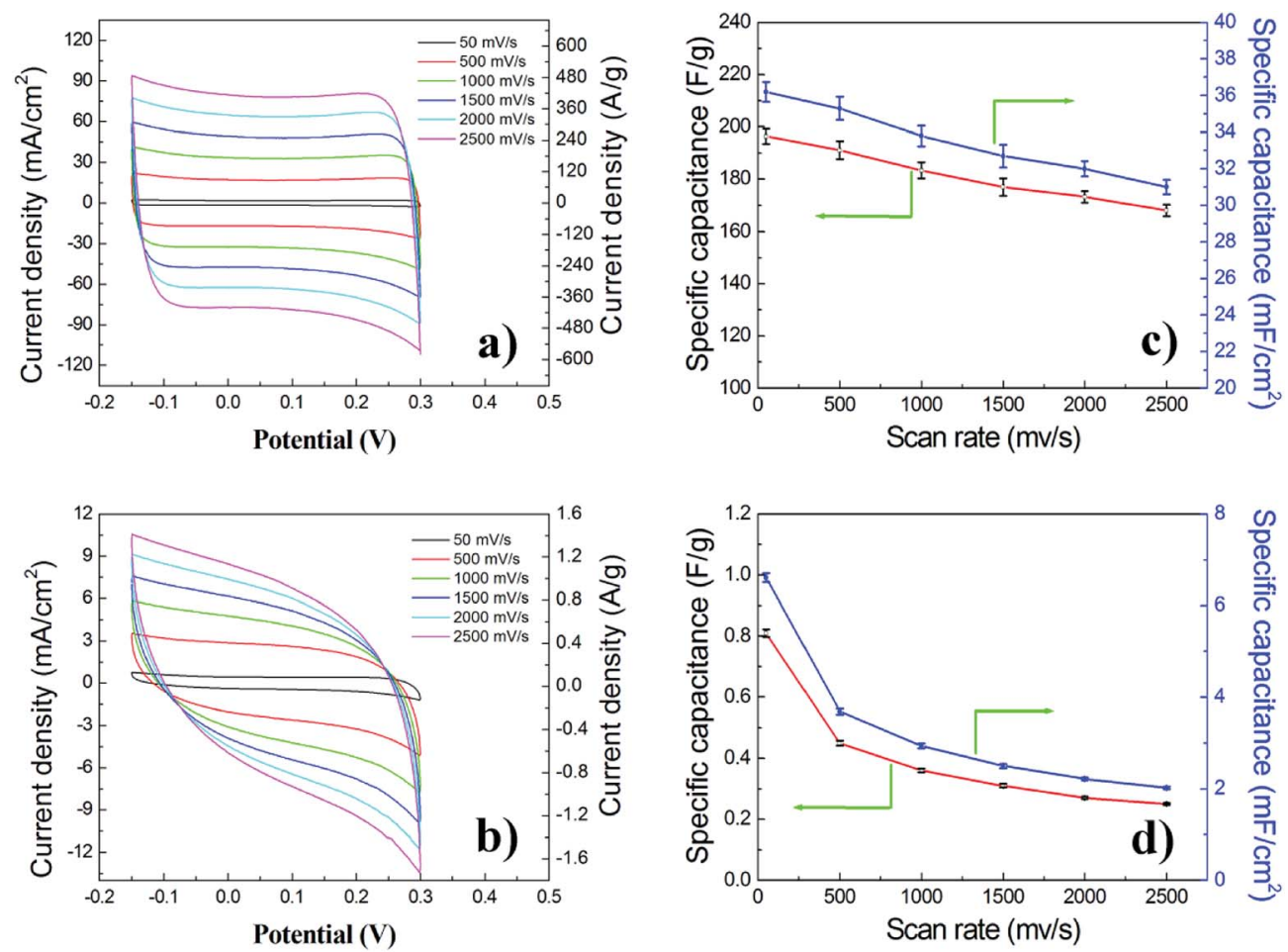

Fig. $6 \mathrm{CV}$ curves collected at scan rates vary from 50 to $2500 \mathrm{mV} \mathrm{s}^{-1}$ in $6 \mathrm{M} \mathrm{KOH}$ for GNSs@Ni electrode (a and c) and FGP@Ni electrode (b and d). The variation of mass specific capacitance and area specific capacitance with scan rates for GNSs@Ni electrode (c) and FGP@Ni electrode (d).

The typical mass capacitance $\left(C_{\mathrm{m}}\right)$ and area capacitance $\left(C_{\mathrm{s}}\right)$ of the electrodes can be calculated according to the following eqn (1) and (2) from CV curves. ${ }^{41}$

$$
\begin{aligned}
& C_{\mathrm{m}}\left(\mathrm{F} \mathrm{g}^{-1}\right)=\frac{1}{2 m v \Delta V} \int_{V_{-}}^{V_{+}} I \mathrm{~d} V \\
& C_{\mathrm{s}}\left(\mathrm{F} \mathrm{cm}^{-2}\right)=\frac{1}{2 S v \Delta V} \int_{V_{-}}^{V_{+}} I \mathrm{~d} V
\end{aligned}
$$

where $I(\mathrm{~A})$ is the response current, $\Delta V(\mathrm{~V})$ is the range of potential, $v\left(\mathrm{~V} \mathrm{~s}^{-1}\right)$ is the potential scan rate, $m(\mathrm{~g})$ is the mass of the active materials in the electrodes, $S\left(\mathrm{~cm}^{2}\right)$ is the area of the electrode. Based on the dependence of mass specific capacitance and area specific capacitance on voltage scan rate (Fig. 6c and d), the $C_{\mathrm{m}}$ and $C_{\mathrm{s}}$ of GNSs@Ni are estimated to be $196.4 \mathrm{~F}$ $\mathrm{g}^{-1}$ and $36.2 \mathrm{mF} \mathrm{cm}^{-2}$ at a scan rate of $50 \mathrm{mV} \mathrm{s}^{-1}$, while they are only $0.81 \mathrm{~F} \mathrm{~g}^{-1}$ and $6.61 \mathrm{mF} \mathrm{cm} \mathrm{cm}^{-2}$ for FGP@Ni electrode. Although the mass loading of FGP@Ni is 28.9 times than of GNSs@Ni, the $C_{\mathrm{m}}$ and $C_{\mathrm{s}}$ of FGP@Ni, respectively, are only $0.41 \%$ and $18.2 \%$ of GNSs@Ni, indicating that the GNSs obtained by electrochemical stripping have a very high specific surface area and a large capacitance capacity. Even at the high scan rate of $2500 \mathrm{mV} \mathrm{s}^{-1}$ the specific capacitance of the capacitor still shows a retention of $85.6 \%\left(168 \mathrm{~F} \mathrm{~g}^{-1}\right)$, which could
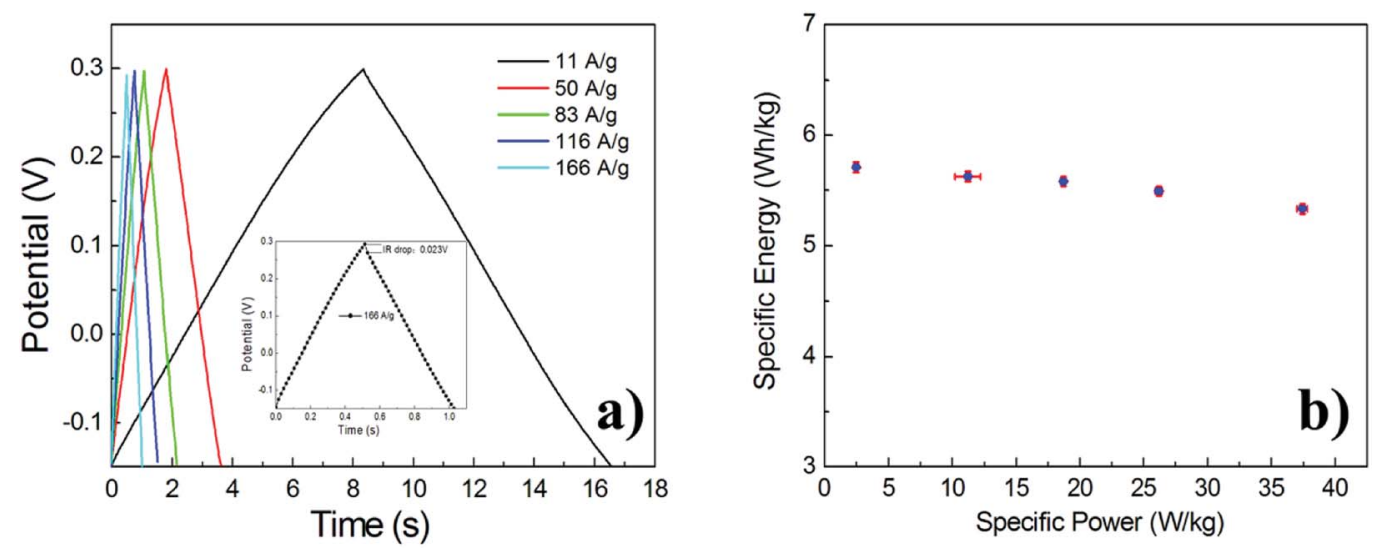

Fig. 7 GCD curves of the GNSs@Ni electrode at different current densities (inset: GCD curves at a current density of $166 \mathrm{~A} \mathrm{~g}^{-1}$ ) (a). Ragone plots of GNSs@Ni electrode (b) 


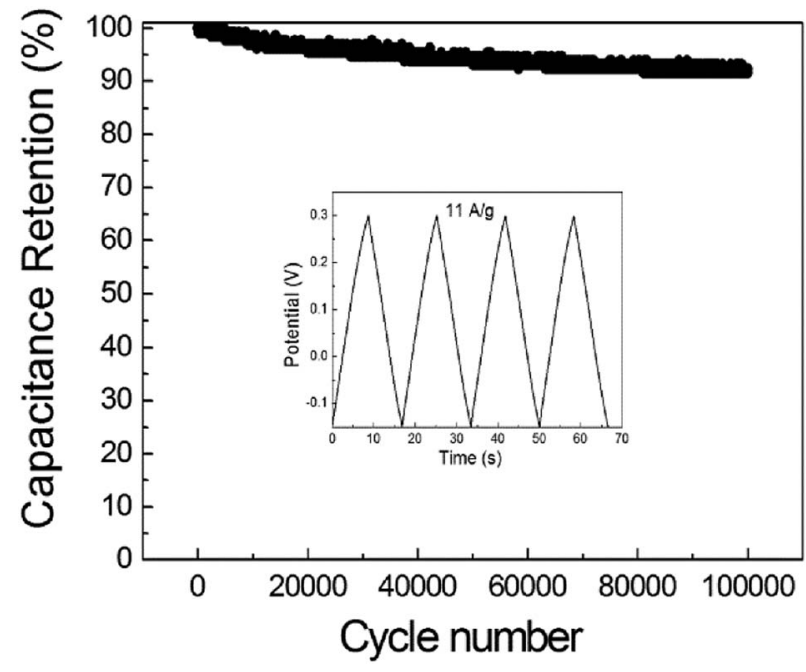

Fig. 8 Cycle stability of the GNSs@Ni electrode (inset: GCD curves at a current density of $11 \mathrm{~A} \mathrm{~g}^{-1}$ ).

owe to the high conductivity and excellent diffusion pathways for electrolyte provided by the unique three dimensional GNSs@Ni all-in-one structure.

As shown in Fig. 7a, Galvanostatic Charge-Discharge (GCD) curves were recorded at different current density, exhibiting good symmetry during charge-discharge. IR drop (about 0.023 v) of GNSs@Ni electrode is observed at a current density of $166 \mathrm{~A} \mathrm{~g}^{-1}$ (inset in Fig. 7a). The typical mass capacitance $\left(C_{\mathrm{m}}\right)$ based on the Galvanostatic Charge-Discharge (GCD) curves were recorded at different current density (Fig. S2 $\dagger$ ). The GNSs showed a superior specific capacitance $\left(202.9 \mathrm{~F} \mathrm{~g}^{-1}\right.$ and $189.5 \mathrm{~F}$ $\mathrm{g}^{-1}$ at $11 \mathrm{~A} \mathrm{~g}^{-1}$ and $166 \mathrm{~A} \mathrm{~g}^{-1}$ ) compared with previously reported literature values. ${ }^{27-29,42}$ Based on the GCD curves, power density $(P)$ and the energy density $(E)$ of the electrode can be calculated through the following eqn (3) and (4). ${ }^{41}$

$$
E\left(\mathrm{~W} \mathrm{~h} \mathrm{~kg}^{-1}\right)=\frac{1}{2} I t \Delta V / m
$$

$$
P\left(\mathrm{~W} \mathrm{~kg}^{-1}\right)=\frac{E}{t}
$$

where $t(\mathrm{~h})$ is the discharge time, $m(\mathrm{~kg})$ is the mass of the active materials in the electrodes, $\Delta V(\mathrm{~V})$ is the range of potential, $I(\mathrm{~A})$ is the discharge current. The energy and power density of the electrode are given by a Ragone plot in Fig. 7b. The asprepared GNSs@Ni electrode exhibits excellent high power performance. As the power density increases from 2.49 to 37.4 $\mathrm{kW} \mathrm{kg}^{-1}$, the energy density of GNSs@Ni decays only $6.5 \%$.

Furthermore, the GNSs@Ni electrode holds 91.5\% capacitance retention after 100000 cycles of GCD measurements at a current density of $11 \mathrm{~A} \mathrm{~g}^{-1}$ (Fig. 8), which reveals remarkable electrochemical stability. To further confirm the superior power performance of the GNSs electrode, electrochemical impedance spectroscopy (EIS) of GNSs@Ni electrode shows pure capacitive behavior even at high frequencies $(215 \mathrm{~Hz})$, which can be attributed to the highly accessible surface of the GNSs (Fig. 9a). ${ }^{35}$ The equivalent series resistance obtained from the intercept of the plot is only $1.28 \Omega \mathrm{cm}^{-2}$ (inset in Fig. 9a), which benefits from the low internal resistance of the electrode. The dependence of phase angle on the frequency for the GNSs@Ni shows that the phase angles are close to $-90^{\circ}$ at low frequencies, revealing an ideal capacitive behavior (Fig. 9b). The characteristic frequency $f_{0}$ at the phase angle of $-45^{\circ}$ is expressed as the point where the capacitive impedance is the same with the resistive impedance. ${ }^{43}$ The GNSs@Ni electrode exhibits an characteristic frequency $\left(f_{0}\right)$ of $7.09 \mathrm{~Hz}$, which corresponds to a time constant $\tau_{0}\left(\tau_{0}=1 / f_{0}\right)$ of $0.14 \mathrm{~s}$, greatly lower than those of conventional carbon-based electrode $\left(\begin{array}{lll}10 & \mathrm{~s}\end{array}\right){ }^{44}$ This rapid frequency response further suggests that the ion transport rate within the GNSs@Ni electrodes were significantly enhanced.

\section{Conclusions}

Through a facile and scalable method, freestanding GNSs@Ni electrodes were fabricated by in situ electrochemical exfoliation of the FGP@Ni electrodes. The binder-free all-in-one structure of the GNSs@Ni electrode inhibited aggregation of graphene
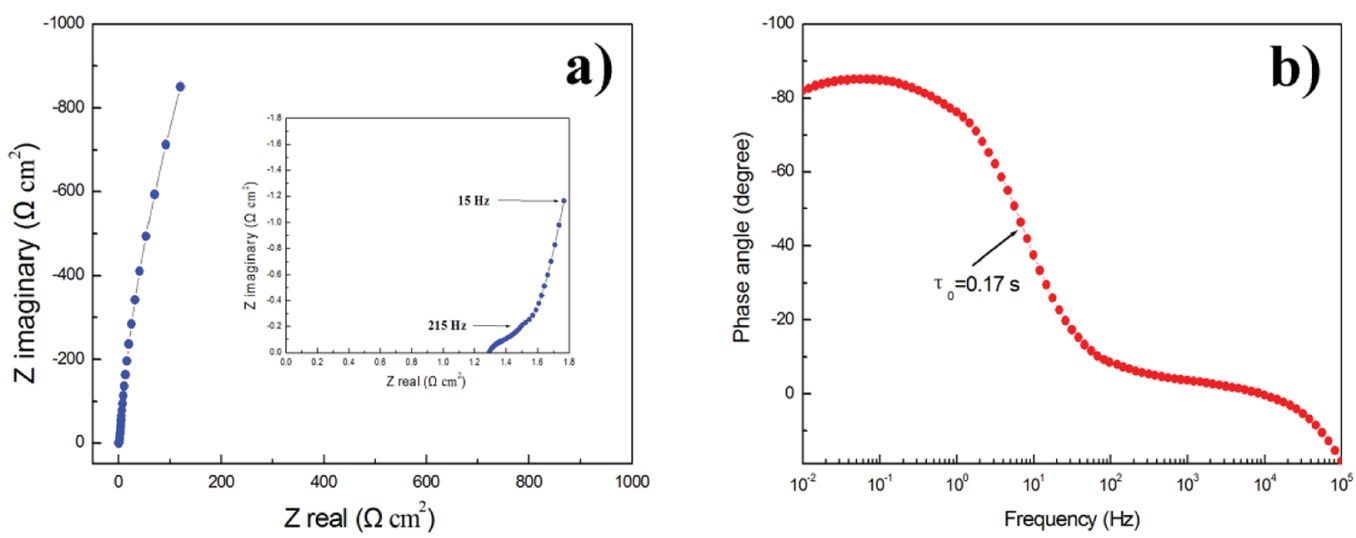

Fig. 9 Nyquist plots of GNSs@Ni electrode (inset: the close-up view of the high-frequency regime) (a). Bode plots of phase angle versus frequency (b). 
nanosheets. Meanwhile, the high electrical conductivity, surface area and mechanical strength were simultaneously achieved in this GNSs@Ni electrode. The experimental results of the prepared electrode exhibited excellent cycling stability, high power density, and high specific capacitance, which shows great potential for the future applications in portable electronics and energy-related device. Meanwhile, this approach for the fabrication of metal integrated three dimensional graphene nanosheets is not only restricted to the electrodes for EDLCs, but provides new insights on developing high-performance hybrid nanoarchitectures electrodes using the prepared graphene nanosheets as the support, which can be widely applied in the fields of electrochemical adsorption, electrochemical catalysis, electrochemical sensing, energy conversion and storage etc.

\section{Conflicts of interest}

There are no conflicts to declare.

\section{Acknowledgements}

This work was supported by the National Natural Science Foundation of China (No. 51702279), the Open Project of State Key Laboratory of Supramolecular Structure and Materials (No. sklssm201835), the Open Project of State Key Laboratory of Heavy Oil Processing (No. sklhop201805) and Natural Science Research Project of Jiangsu Higher Education Institutions (No. 18KJB430029).

\section{References}

1 M. Winter and R. J. Brodd, What Are Batteries, Fuel Cells, and Supercapacitors?, Chem. Rev., 2004, 104, 4245.

2 B. E. Conway, Transition from supercapacitor to battery behavior in electrochemical energy storage, J. Electrochem. Soc., 1991, 138, 1539.

3 P. Simon, Y. Gogotsi and B. Dunn, Where do batteries end and supercapacitors begin?, Science, 2014, 343, 1210.

4 B. Huang, X. D. Zheng, X. P. Fan, G. H. Song and M. Lu, Enhanced rate performance of nano-micro structured $\mathrm{LiFePO}_{4} / \mathrm{C}$ by improved process for high-power Li-ion batteries, Electrochim. Acta, 2011, 56, 4865.

5 J. Azadmanjiri, V. K. Srivastava, P. Kumar, M. Nikzad, J. Wang and A. Yu, Two- and three-dimensional graphenebased hybrid composites for advanced energy storage and conversion devices, J. Mater. Chem. A, 2018, 6, 702.

6 L. Jiang, L. Sheng, C. Long, T. Wei and Z. Fan, Functional pillared graphene frameworks for ultrahigh volumetric performance supercapacitors, Adv. Energy Mater., 2015, 5, 1500771.

7 Z. Gao, C. Chen, J. Chang, L. Chen, D. Wu, F. Xu and K. Jiang, Balanced energy density and power density: asymmetric supercapacitor based on activated fullerene carbon soot anode and graphene- $\mathrm{Co}_{3} \mathrm{O}_{4}$ composite cathode, Electrochim. Acta, 2018, 260, 932.
8 A. González, E. Goikolea, J. A. Barrena and R. Mysyk, Review on supercapacitors: technologies and materials, Renewable Sustainable Energy Rev., 2016, 58, 1189.

9 Y. Wang, Y. Song and Y. Xia, Electrochemical capacitors: mechanism, materials, systems, characterization and applications, Chem. Soc. Rev., 2016, 45, 5925.

10 D. Y. Lee, G. H. An and H. J. Ahn, High-surface-area tofu based activated porous carbon for electrical double-layer capacitors, J. Ind. Eng. Chem., 2017, 52, 121.

11 K. Zhu, Y. Wang, J. A. Tang, S. Guo, Z. Gao, Y. Wei, G. Chen and Y. Gao, A high-performance supercapacitor based on activated carbon fibers with an optimized pore structure and oxygen-containing functional groups, Mater. Chem. Front., 2017, 1, 958.

12 Y. Huang, Y. Zhao, Q. Gong, M. Weng, J. Bai, X. Liu, Y. Jiang, J. Wang, D. Wang, Y. Shao, M. Zhao, D. Zhuang and J. Liang, Experimental and correlative analyses of the ageing mechanism of activated carbon based supercapacitor, Electrochim. Acta, 2017, 228, 214.

13 B. Li, F. Dai, Q. Xiao, L. Yang, J. Shen, C. Zhang and M. Cai, Activated carbon from biomass transfer for high-energy density lithium-ion supercapacitors, Adv. Energy Mater., 2016, 6, 1600802.

14 L. Liu, Z. Niu and J. Chen, Flexible supercapacitors based on carbon nanotubes, Chin. Chem. Lett., 2018, 29, 571.

15 A. Izadi-Najafabadi, S. Yasuda, K. Kobashi, T. Yamada, D. N. Futaba, H. Hatori, M. Yumura, S. Lijima and K. Hata, Extracting the full potential of single-walled carbon nanotubes as durable supercapacitor electrodes operable at $4 \mathrm{~V}$ with high power and energy density, Adv. Mater., 2010, 22, E235.

16 J. Liu, F. Mirri, M. Notarianni, M. Pasquali and N. Motta, High performance all-carbon thin film supercapacitors, $J$. Power Sources, 2015, 274, 823.

17 M. Li, L. Zong, X. Li, J. You, X. Wu, Q. Kong and C. Li, Fibrous carbon nanosheets from Kevlar nanofibrils: compromising one and two dimensions of carbon nanomaterials for optimal capacitive performance, Carbon, 2017, 123, 565.

18 D. He, J. Niu, M. Dou, J. Ji, Y. Huang and F. Wang, Nitrogen and oxygen co-doped carbon networks with a mesoporedominant hierarchical porosity for high energy and power density supercapacitors, Electrochim. Acta, 2017, 238, 310.

19 X. Wang, H. Ma, X. He, J. Wang, J. Han and Y. Wang, Fabrication of interconnected mesoporous carbon sheets for use in high-performance supercapacitors, New Res. Carbon Mater., 2017, 32, 213.

$20 \mathrm{M}$. Yang and Z. Zhou, Recent breakthroughs in supercapacitors boosted by nitrogen-rich porous carbon materials, Adv. Sci., 2017, 4, 1600408.

21 R. Liu, L. Ma, J. Mei, S. Huang, S. Yang, E. Li and G. Yuan, Large areal mass, mechanically tough and freestanding electrode based on heteroatom-doped carbon nanofibers for flexible supercapacitors, Chem.-Eur. J., 2017, 23, 2610.

22 J. Huang, Y. Liang, H. Hu, S. Liu, Y. Cai, H. Dong, M. Zheng, Y. Xiao and Y. Liu, Ultrahigh-surface-area hierarchical porous carbon from chitosan: acetic acid mediated 
efficient synthesis and its application in superior supercapacitors, J. Mater. Chem. A, 2017, 5, 24775.

23 M. Anouti, E. Couadou, L. Timperman and H. Galiano, Protic ionic liquid electrolyte for high-densities electrochemical capacitors with activated carbon, Electrochim. Acta, 2012, 64, 110.

24 Y. L. Shi, M. Jin, Y. Zhang, Y. B. Niu, J. C. Gao and C. M. Li, Chemically exfoliating biomass into a graphene-like porous active carbon with rational pore structure, good conductivity, and large surface area for high-performance supercapacitors, Adv. Energy Mater., 2017, 8, 1702545.

25 L. Wu, W. Li, P. Li, S. Liao, S. Qiu, M. Chen, Y. Guo, Q. Li, C. Zhu and L. Liu, Powder, paper and foam of few-layer graphene prepared in high yield by electrochemical intercalation exfoliation of expanded graphite, Small, 2014, 10, 1421.

26 D. Sun, X. Yan, J. Lang and Q. Xue, High performance supercapacitor electrode based on graphene paper via flame-induced reduction of graphene oxide paper, J. Power Sources, 2013, 222, 52.

27 F. H. Kuok, H. H. Chien, C. C. Lee, Y. C. Hao, I. S. Yu, C. C. Hsu, I. C. Cheng and J. Z. Chen, Atmosphericpressure-plasma-jet processed carbon nanotube (CNT)reduced graphene oxide (rGO) nanocomposites for gelelectrolyte supercapacitors, RSC Adv., 2018, 8, 2851.

28 L. Gao, Z. Zhang, J. Zhao, J. Zhou, Z. Miao, W. Si and S. Zhuo, Controllable synthesis of graphene scrolls and their performance for supercapacitors, RSC Adv., 2018, 8, 19164.

29 P. Chen, J. J. Yang, S. S. Li, Z. Wang, T. Y. Xiao, Y. H. Qian and S. H. Yu, Hydrothermal synthesis of macroscopic nitrogen-doped graphene hydrogels for ultrafast supercapacitor, Nano Energy, 2013, 2, 249.

30 Z. Xiong, C. Liao and X. Wang, A self-assembled macroporous coagulation graphene network with high specific capacitance for supercapacitor applications, $J$. Mater. Chem. A, 2014, 2, 19141.

31 X. Cao, Z. Yin and H. Zhang, Three-dimensional graphene materials: preparation, structures and application in supercapacitors, Energy Environ. Sci., 2014, 7, 1850.

32 R. S. Dey, H. A. Hjuler and Q. Chi, Approaching the theoretical capacitance of graphene through copper foam integrated three dimensional graphene networks, J. Mater. Chem. A, 2015, 3, 6324.

33 K. Shu, C. Wang, S. Li, C. Zhao, Y. Yang, H. Liu and G. Wallace, Flexible free-standing graphene paper with interconnected porous structure for energy storage, $J$. Mater. Chem. A, 2015, 3, 4428.

34 M. F. El-Kady and R. B. Kaner, Scalable fabrication of highpower graphene micro-supercapacitors for flexible and onchip energy storage, Nat. Commun., 2013, 4, 1475.

35 H. Huang, L. Xu, Y. Tang, S. Tang and Y. Du, Facile synthesis of nickel network supported three-dimensional graphene gel as a lightweight and binder-free electrode for high rate performance supercapacitor application, Nanoscale, 2014, 6, 2426.

36 J. Hao, Y. Zhong, Y. Liao, D. Shu, Z. Kang, X. Zou, C. He and S. Guo, Face-to-face self-assembly graphene $/ \mathrm{MnO}_{2}$ nanocomposites for supercapacitor applications using electrochemically exfoliated graphene, Electrochim. Acta, 2015, 167, 412.

37 W. Chen, R. B. Rakhi, L.-B. Hu, X. Xie, Y. Cui and H. N. Alshareef, High-performance nanostructured supercapacitors on a sponge, Nano Lett., 2011, 11, 5165.

38 L. Zhang, J. Wang, J. Zhu, X. Zhang, K. S. Hui and K. N. Hui, $3 \mathrm{D}$ porous layered double hydroxides grown on graphene as advanced electrochemical pseudocapacitor materials, $J$. Mater. Chem. A, 2013, 1, 9046.

39 S. Jiang, T. Shi, X. Zhan, H. Long, S. Xi, H. Hu and Z. Tang, High-performance all-solid-state flexible supercapacitors based on two-step activated carbon cloth, J. Power Sources, 2014, 272, 16.

40 A. C. Ferrari, J. C. Meyer, V. Scardaci, C. Casiraghi, M. Lazzeri, F. Mauri, S. Piscanec, D. Jiang, K. S. Novoselov, S. Roth and A. K. Geim, Raman spectrum of graphene and graphene layers, Phys. Rev. Lett., 2006, 97, 187401.

41 L. Wang, Y. Zheng, Q. Zhang, L. Zuo, S. Chen, S. Chen, H. Hou and Y. Song, Template-free synthesis of hierarchical porous carbon derived from low-cost biomass for high performance supercapacitors, $R S C A d v$., 2014, 4, 51072.

42 B. You, J. Jiang and S. Fan, Three-dimensional hierarchically porous all-carbon foams for supercapacitor, ACS Appl. Mater. Interfaces, 2014, 6, 15302.

43 Y. Xu, Z. Lin, X. Zhong, X. Huang, N. O. Weiss, Y. Huang and $X$. Duan, Holey graphene frameworks for highly efficient capacitive energy storage, Nat. Commun., 2014, 5, 4554.

44 M. F. El-Kady, V. Strong, S. Dubin and R. B. Kaner, Laser scribing of high performance and flexible graphene-based electrochemical capacitors, Science, 2012, 335, 1326. 